\title{
Dexamethasone as a chemosensitizer for breast cancer chemotherapy: Potentiation of the antitumor activity of adriamycin, modulation of cytokine expression, and pharmacokinetics
}

\author{
HUI WANG ${ }^{1,2,4}$, YING WANG ${ }^{1}$, ELIZABETH R. RAYBURN ${ }^{1}$, DONALD L. HILL ${ }^{1}$, \\ JOHN J. RINEHART ${ }^{5}$ and RUIWEN ZHANG ${ }^{1-3}$ \\ ${ }^{1}$ Division of Clinical Pharmacology, Department of Pharmacology and Toxicology, ${ }^{2}$ Cancer Pharmacology \\ Laboratory, Comprehensive Cancer Center, ${ }^{3}$ Gene Therapy Center, University of Alabama \\ at Birmingham,Birmingham, AL, USA; ${ }^{4}$ Institute for Nutritional Sciences, Shanghai Institutes \\ for Biological Sciences (SIBS), Chinese Academy of Sciences, Shanghai 200031, P.R. China; \\ ${ }^{5}$ University of Kentucky Markey Cancer Center, Lexington, KY 40536, USA
}

Received May 5, 2006; Accepted July 17, 2006

\begin{abstract}
Dexamethasone (DEX) is mainly used as an antiemetic agent in cancer therapy. We have recently demonstrated that DEX pretreatment increases the antitumor activity of the cancer chemotherapeutic agents carboplatin and gemcitabine, and decreases host toxicity in nude mouse xenograft models of human cancer. However, the underlying mechanisms are not fully understood. The present study was designed to determine the effects of DEX pretreatment on the anticancer activity of adriamycin (ADR) in a syngeneic model of breast cancer (4T1), emphasizing the effects of DEX on cytokine expression and modulation of ADR pharmacokinetics. We have demonstrated five major new findings about DEX pretreatment: a) it enhances the therapeutic effect of ADR, inducing almost complete inhibition of tumor growth; b) it increases tumor ADR accumulation; c) it modulates the expression of cytokines produced by the tumor, increasing TNF $\alpha$ and decreasing IL-1B and VEGF expression; d) it enhances the effects of ADR on induction of apoptosis and inhibition of cell proliferation; and e) it suppresses nuclear NFKB activation and inhibits ADR-induced NFKB activation, possibly via IкB up-regulation. These findings suggest that
\end{abstract}

Correspondence to: Dr Hui Wang, Department of Pharmacology and Toxicology, 1670 University Blvd., Room 112, University of Alabama at Birmingham, Birmingham, AL 35294-0019, USA

E-mail: hui.wang@ccc.uab.edu.

Abbreviations: DEX, dexamethasone; ADR, adriamycin; FBS, fetal bovine serum; PBS, phosphate-buffered saline; HPLC, high performance liquid chromatography; TNF $\alpha$, tumor necrosis factor $\alpha$; IL-1ß, interleukin 1B; VEGF, vascular endothelial growth factor; $\mathrm{NF} \kappa \mathrm{B}$, nuclear factor kappa $\mathrm{B}$; IкB, $\mathrm{NF}_{\kappa} \mathrm{B}$ inhibitory protein; IFP, interstitial fluid pressure

Key words: dexamethasone, adriamycin, pro-inflammatory cytokine, NFkB
DEX can be used as a chemosensitizer and chemoprotectant. These results provide a rationale for the expanded clinical use of DEX for cancer therapy.

\section{Introduction}

Although early detection and improvements in surgery, chemotherapy, and radiation therapy have led to increased survival of patients with breast cancer, it remains the second cause of cancer death in women in North America (1). Additionally, although there have been improvements, patient quality of life is still an issue due to the side effects of many of the agents used for therapy. Therefore, increasing efforts need to be devoted to the development of more effective, less toxic therapeutic approaches.

Among therapeutic agents used for breast cancer, adriamycin (ADR) remains a primary chemotherapeutic agent, achieving response rates of $40-50 \%$ as a single agent and $60-80 \%$ in combination $(2,3)$. For example, the combination of ADR with cyclophosphamide and 5fluorouracil (5-FU) leads to response rates of $50-80 \%$ (3). Unfortunately, as is seen with other DNA-interactive chemotherapeutic agents, ADR causes various side effects, among which myelosuppresion is one of the most serious. Several bone marrow protective and stimulatory agents, such as granulocyte colony stimulating factor (G-CSF), have been applied following chemotherapy to reduce the associated hematotoxicity.

Inflammatory responses are believed to play an important role in tumor initiation, progression, and metastasis $(4,5)$. Pro-inflammatory cytokines, such as tumor necrosis factor (TNF) $\alpha$, interleukin (IL)-1, and IL-6, are produced not only by inflammatory cells but also by tumor and stromal cells $(4,5)$. Acting as essential communicating molecules between tumor and inflammatory cells, pro-inflammatory cytokines promote tumor cell proliferation and progression. Dexamethasone (DEX), a synthetic glucocorticoid, is widely used in autoimmune diseases as an anti-inflammatory agent and 
immunosuppressant, mainly due to its capacity to inhibit the production of pro-inflammatory cytokines $(6,7)$. DEX is also clinically used in the treatment of human malignancies, especially leukemia and myelomas. It has also been given during and after chemotherapy for patients with solid tumors (including breast cancer) to prevent nausea and vomiting and reduce acute toxicity (8-11).

We recently demonstrated that pretreatment with DEX decreased host tissue uptake of carboplatin and gemcitabine, indicating a chemoprotective action of DEX (12). Furthermore, DEX pretreatment enhanced the anticancer effects of carboplatin and gemcitabine in several human cancer in vivo models, including those of colon, lung, breast, and brain cancers, indicating that DEX may act as a chemosensitizer for cancer chemotherapy (13). The mechanisms responsible for the chemoprotective effects of DEX, however, are not fully understood. One possibility is that DEX exerts its effects through the suppression of inflammatory responses that occur during tumor development in the host. Mechanistic studies have shown that DEX prevents NFkB activation via increasing IкB synthesis, and that this action depends on the early and transient activation of NFKB $(14,15)$. Our present work focused on investigation of the effects of DEX pretreatment on breast cancer chemotherapy, as well as mechanisms responsible for these effects. Our hypothesis was that pretreatment with DEX may affect expression of proinflammatory cytokines through regulation of the NFKB pathway, thereby enhancing the effects of chemotherapeutic agents in the treatment of breast cancer. This could potentially lead to a change in the clinical use of DEX, possibly altering the administration schedule, and indicating that use of DEX may be preferential to other anti-nausea and hematoprotective agents because of its numerous other protective effects.

\section{Materials and methods}

Chemicals and reagents. All chemicals and solvents were high-performance liquid chromatography (HPLC) grade or certified grade. Methanol, acetonitrile and ammonium formate were purchased from Fisher Chemicals (Atlanta, GA). DEX (clinical grade) was purchased from American Regent Laboratories (Shirley, NJ), and ADR (doxorubicin hydrochloride injection) was purchased from NovaPlus. Centrifree micropartition systems (catalog no. 4104) were purchased from Millipore Corp. (Bedford, MA). Cell culture media, fetal bovine serum, sodium pyruvate, nonessential amino acids, penicillin-streptomycin, and other cell culture supplies were provided by the Comprehensive Cancer Center Media Preparation Shared Facility, University of Alabama at Birmingham. Tissue solubilizer (TS-2) was purchased from Research Products Inc. (Mount Prospect, IL).

Animals. The protocol for animal care and use was approved by the Institutional Animal Care and Use Committee of the University of Alabama at Birmingham. Female BALB/c mice (4-6 weeks of age) were obtained from Frederick Cancer Research Facility (Frederick, MD). For 1 week before the study, all animals were fed with commercial diet and provided water ad libitum.
Cell culture. The mouse mammary cancer cell line 4T1 was obtained from the ATCC and cultured according to their instructions. The culture medium contained 90\% DMEM, $10 \%$ fetal bovine serum, $1 \%$ penicillin-streptomycin, $1 \mathrm{mM}$ sodium pyruvate, and $1 \mathrm{mM}$ non-essential amino acids. Cells were incubated at $37^{\circ} \mathrm{C}$ in $5 \% \mathrm{CO}_{2}$.

Animal syngeneic tumor model. The mouse mammary cancer model was established in female BALB/c mice. When cultured 4T1 cells in monolayers reached a confluence of $80 \%$, cells were trypsinized, harvested by centrifugation, washed with the serum-free DMEM medium, and resuspended in the same medium. The cell suspension was then injected subcutaneously (s.c.) $\left(10^{5}\right.$ cells; total volume, $\left.0.2 \mathrm{ml}\right)$ into the left inguinal area of 5 to 7-week-old female BALB/c mice. The animals were monitored for activity, physical condition, body weight, and tumor growth. Tumor growth was determined by caliper measurement in two perpendicular diameters of the implant. As reported previously $(13,16-18)$, tumor mass (in g) was calculated by the formula $1 / 2 \mathrm{a} \mathrm{x} \mathrm{b}^{2}$, where $\mathrm{a}$ is the long diameter, and $\mathrm{b}$ is the short diameter (in $\mathrm{cm}$ ).

In vivo chemotherapy. The mice bearing $4 \mathrm{~T} 1$ tumors were randomly divided into various treatment and control groups (5 mice/group). Animals were treated with DEX by s.c. injection at a dose of $0.1 \mathrm{mg} /$ day for 5 days or with saline (as controls) before chemotherapy (day -4 to 0 ). On day 0 , ADR was administered intravenously (i.v.) at a single, clinically relevant dose of $10 \mathrm{mg} / \mathrm{kg}\left(30 \mathrm{mg} / \mathrm{m}^{2}\right)$.

Pharmacokinetics of adriamycin. The 4T1 tumor-bearing mice were pretreated with DEX (s.c., $0.1 \mathrm{mg}$ /day/mouse for 5 days) or saline (as controls) and, $1 \mathrm{~h}$ after the fifth dose of DEX, were given a single i.v. bolus administration of ADR $(10 \mathrm{mg} / \mathrm{kg})$ via a tail vein. At various times, blood samples were collected in heparinized tubes, and plasma was separated by centrifugation at $20,000 \mathrm{x}$ g for $5 \mathrm{~min}$. Tissues were collected at various times and immediately blotted on Whatman No. 1 filter paper, trimmed of extraneous fat or connective tissue, weighed, and homogenized in physiological saline $(0.9 \% \mathrm{NaCl}, 5 \mathrm{ml} / \mathrm{g}$ wet weight $)$. The resulting homogenates were stored at $-70^{\circ} \mathrm{C}$ until further analysis.

HPLC analysis of adriamycin in plasma and tissues. Plasma or tissue samples $(200 \mu \mathrm{l})$ were added to $400 \mu \mathrm{l}$ of acetonitrile and mixed thoroughly by vortexing for $1 \mathrm{~min}$. After centrifugation at $14,000 \times \mathrm{g}$ for $10 \mathrm{~min}$, the organic layer was removed to a glass test tube and evaporated by air at room temperature. The residue was reconstituted in $300 \mu 1$ of mobile phase that consisted of $0.1 \mathrm{M}$ ammonium formate ( $\mathrm{pH} 4.0)$ and acetonitrile (85:15), vortexed thoroughly, and filtered through a $0.2-\mu \mathrm{m}$ polypropylene syringe filter for HPLC injection. ADR levels in plasma and tissue samples were determined by a method previously reported (19) with some modifications. Briefly, the mobile phase, which consisted of $0.1 \mathrm{M}$ formate buffer and acetonitrile, was used in a linear gradient from $85 \% \mathrm{~A}$ (ammonium formate) and $15 \% \mathrm{~B}$ (acetonitrile) to $70 \% \mathrm{~A}$ and $30 \% \mathrm{~B}$ up to $6 \mathrm{~min}$, then changed to $50 \% \mathrm{~A} / 50 \% \mathrm{~B}$ within $4 \mathrm{~min}$. This gradient continued to $100 \% \mathrm{~B}$ during $2 \mathrm{~min}$, then returned to $85 \% \mathrm{~A}$ 
and $15 \% \mathrm{~B}$ within an additional $2 \mathrm{~min}$. The flow rate was maintained at $1 \mathrm{ml} / \mathrm{min}$. ADR, eluted from a RP-C ${ }_{18}$ column (ZORBAX SB-C ${ }_{18}, 5 \mu \mathrm{M}, 4.6 \times 150 \mathrm{~mm}$, Agilent), was monitored using a fluorescence detector (Agilent 1050 series) at an excitation wavelength of $480 \mathrm{~nm}$ and an emission wavelength of $550 \mathrm{~nm}$. Quantitation of plasma or tissue ADR was accomplished by use of an external standard curve $(0-5,000 \mathrm{ng} / \mathrm{ml})$ that was prepared on a daily basis. Linear regression and correlation analysis were completed to establish the standard peak-area/concentration curves for ADR.

Real-time quantitative PCR (RT-Q-PCR) for analyses of cytokine mRNA expression. The mRNA levels of three cytokines in tumor tissue, TNF $\alpha$, IL-1ß, and VEGF, were measured by quantitative real-time RT-PCR. A small piece of tumor tissue $\left(9 \mathrm{~mm}^{3}\right)$ was cut and stored in RNAlater (Ambion, Austin, TX) at the time of animal dissection. Total RNA was isolated and purified with the RNeasy Minikit (Qiagen, Valencia, CA). With a TaqMan Reverse Transcription kit (ABI, Foster City, CA), $2 \mu \mathrm{g}$ of total RNA were used to form cDNA in a $25-\mu 1$ solution, following the manufacturer's suggested protocol. Real-time quantitative PCR was accomplished with an ABI PRISM 7900 machine. Assays-on-demand probes and primer master mixes from ABI were used for all reverse transcription (RT)-PCR reactions. The comparative $\mathrm{C}_{\mathrm{T}}$ (threshold cycle) method described in the manufacturer's protocol was used for relative quantitation of expression, comparing treated samples to concordant untreated controls and to the internal control, 18s rRNA. Each sample was run in duplicate.

Cell viability assay. The viability of cultured 4T1 cells was assayed by using the MTT [(tetrazolium salt 3-(4, 5-dimethylthiazol-2-yl)-2,5-diphenyl tetrazolium bromide)] assay as reported previously $(16,17)$. In brief, cells were seeded in 96-well microtiter plates (500-1,000 cells/well) and exposed to DEX for $24 \mathrm{~h}$. ADR was then added to the cells that were incubated for additional $24 \mathrm{~h}$. Fresh media, as well as MTT stock solution, were added to each well, reaching a final MTT concentration of $0.5 \mu \mathrm{g} / \mathrm{ml}$. Cells were cultured for $2-4 \mathrm{~h}$ to allow color development and, at that time, DMSO was added to the wells to solubilize the formazan products. Absorbances were measured at $570 \mathrm{~nm}$.

Cell proliferation assay. Cell proliferation was detected by labeling cells with BrdU using a cell proliferation assay kit (Oncogene Science, San Diego, CA) as reported previously (16-18). After the same treatment protocol described in the MTT assay, cells were incubated with $\operatorname{BrdU}(1: 2,000)$ for $6-8 \mathrm{~h}$ according to the manufacturer's protocol. The incorporated BrdU was detected by monoclonal anti-BrdU antibody via measuring absorbance at dual wavelengths of 450/540 nm with an OPTImax microplate reader (Molecular Devices, Sunnyvale, CA).

Detection of apoptosis. After the same treatment protocol as above, apoptotic cells were analyzed using an annexin V-FITC apoptosis detection kit (BioVision, Mountain View, CA), following the previously reported protocol (16-18). Briefly, total cells collected by centrifugation were resuspended in $500 \mu 1$ of $1 \mathrm{x}$ binding buffer, followed by addition of $5 \mu 1$ of Annexin V-FITC and $5 \mu 1$ of propidium iodide (PI). Samples were incubated in the dark for $5 \mathrm{~min}$, then analyzed by using a Becton Dickinson FACSCalibur (Ex = $488 \mathrm{~nm}$; $\mathrm{Em}=530 \mathrm{~nm}$ ). Each sample was evaluated in duplicate and data were expressed as the percentage of the control.

Western blot analysis. The protein levels of cytoplasmic and nuclear NFкB (p65) and IкB were measured by Western blot analysis as described previously (13). Cells were exposed to DEX either alone or in combination with ADR, followed by fractionation of cytoplasmic and nuclear proteins using nuclear and cytoplasmic extraction reagents from Pierce (Rockford, IL). The polyclonal antibodies against NFkB (p65) and IкB were purchased from Oncogene Research Products (Boston, MA).

$N F \kappa B$-luciferase promoter assay. NFкB-luciferase promoter activity was detected by the Dual-Luciferase ${ }^{\circledR}$ reporter assay system (Promega, Madison, WI) following the manufacturer's protocol. Briefly, 4T1 cells were seeded in a 6-well microtiter plate $\left(10^{4}\right.$ cells/well), then transfected with NFKB-luciferase promoter vector that was kindly provided by Dr Chuanshu Huang, New York University School of Medicine. After 8 h, cells were pre-treated with or without DEX for $24 \mathrm{~h}$, followed by exposure to ADR for an additional $24 \mathrm{~h}$. Cells were then harvested and activity was detected using the Dual-Luciferase ${ }^{\circledR}$ reporter assay system. The results were expressed as NFKB luciferase activity relative to that of the internal control.

\section{Results}

Pretreatment with DEX enhances the therapeutic effects of $A D R$ in vivo. The effect of DEX pretreatment on the antitumor activity of ADR was studied in a 4T1 syngeneic mouse model of breast cancer. The animals were treated with s.c. DEX at a dose of $0.1 \mathrm{mg} /$ day for 5 days (days -4 to 0 ), followed by i.v. ADR (on day 0). As illustrated in Fig. 1A, DEX alone showed a modest inhibitory effect on tumor growth. Pretreatment with DEX, however, enhanced the therapeutic effect of ADR, inducing almost complete inhibition of tumor growth. DEX alone produced no observable toxicity, as determined by clinical inspection and measurement of animal weights as indices of toxicity or side effects. ADR was also well tolerated. Further, DEX pretreatment had no effect on the toxicity profiles of ADR (Fig. 1B).

Pretreatment with DEX increases the accumulation of $A D R$ in tumor tissues in tumor-bearing mice. The time-concentration curves are illustrated in Fig. 2. Although there were no significant differences in plasma pharmacokinetics of ADR between control mice and mice pre-treated with DEX (Fig. 2A), DEX pretreatment significantly increased the ADR concentration in tumor tissue, especially for later time points (4 and $24 \mathrm{~h}$ after dosing) (Fig. 2B). No appreciable differences in the concentrations of ADR were observed in spleen (Fig. 2C), kidney, liver or heart (data not shown).

Pretreatment with DEX influences cytokine expression at the $m R N A$ level in vivo. In the 4T1 tumors, expression levels of 

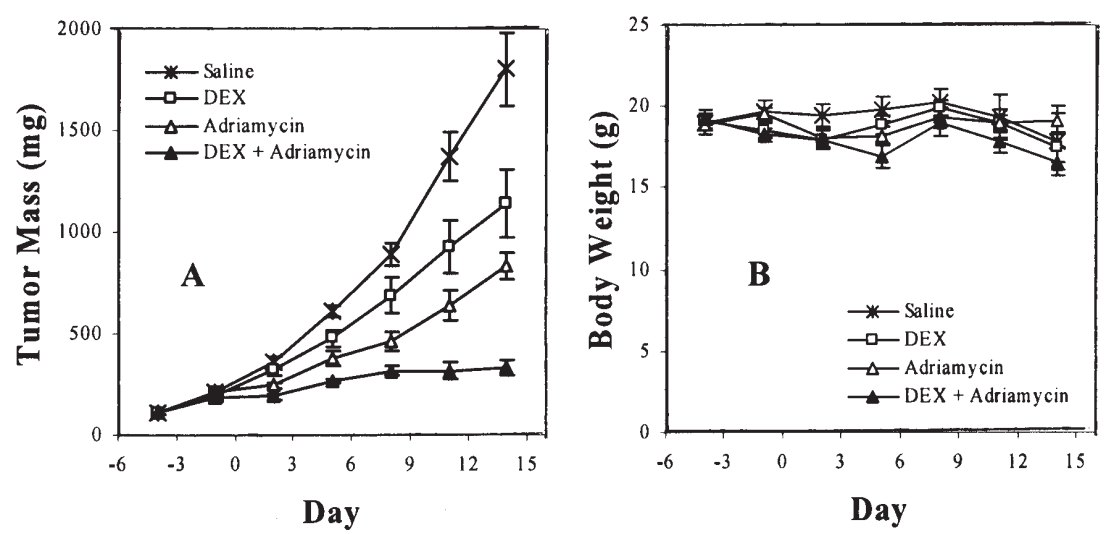

Figure 1. Effects of DEX pretreatment on antitumor activity (A) and body weight (B) following ADR chemotherapy in BALB/c mice bearing 4T1 syngeneic tumors. Animals, randomly divided into various treatment and control groups (5 mice/group), were pretreated with DEX (s.c., $0.1 \mathrm{mg} /$ day for 5 days, days -4 to 0 ) or saline (as control). ADR was administered i.v. at a single dose of $10 \mathrm{mg} / \mathrm{kg}\left(30 \mathrm{mg} / \mathrm{m}^{2}\right)$ on day 0 . Tumor mass was expressed as mean \pm SD.
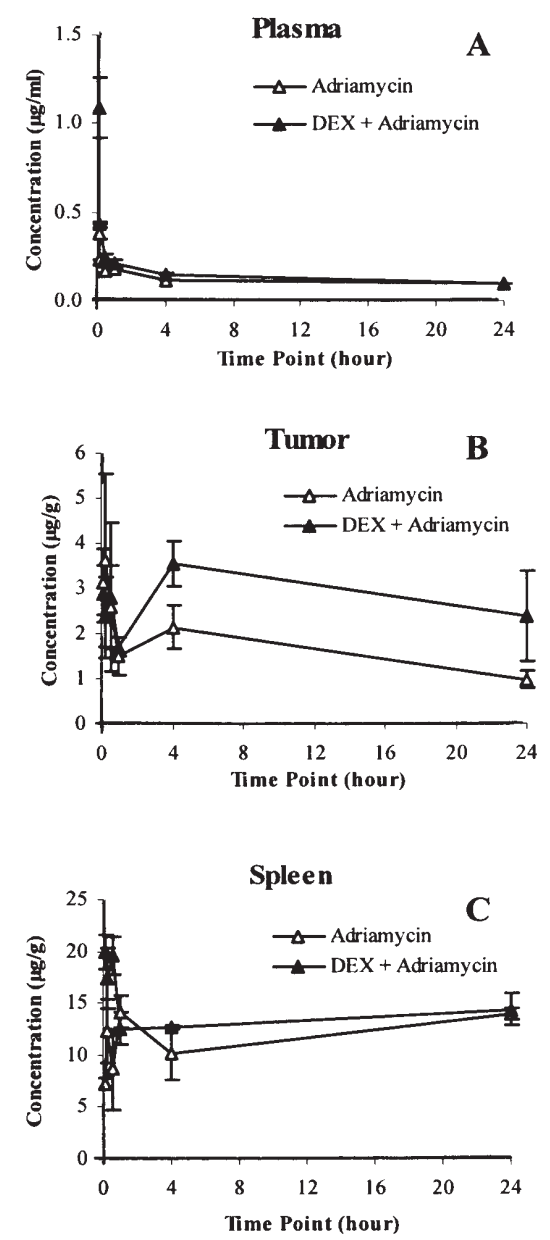

Figure 2. Pharmacokinetics of ADR in BALB/c mice bearing 4T1 tumors. Animals were pretreated with DEX (s.c., $0.1 \mathrm{mg} /$ day/mouse for 5 days) or saline (as controls) and given ADR at a single i.v. dose of $10 \mathrm{mg} / \mathrm{kg}\left(30 \mathrm{mg} / \mathrm{m}^{2}\right)$. Plasma (A), tumor (B) and spleen (C) samples were collected at various times up to $24 \mathrm{~h}$. The ADR levels were analysed by high-performance liquid chromatography.

TNF $\alpha$, IL-1ß and VEGF mRNA were determined by quantitative real-time RT-PCR; the results are shown in Fig. 3. In mice treated with ADR alone, the expression of $\mathrm{TNF} \alpha$ mRNA did not show any appreciable change. Pretreatment with DEX, however, enhanced the expression at 4 and $24 \mathrm{~h}$
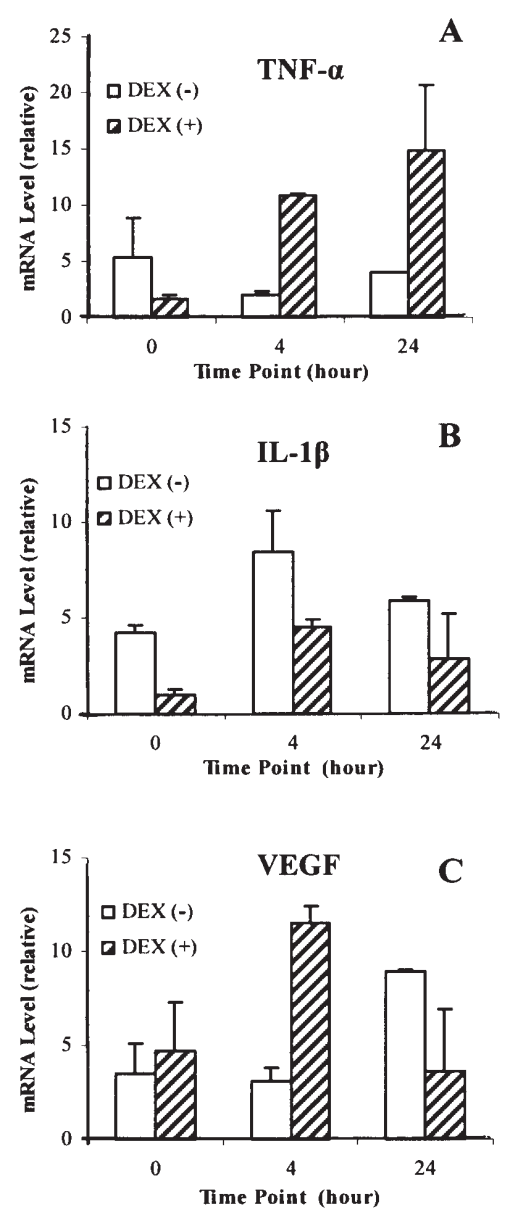

Figure 3. Cytokine mRNA levels in tumor samples of BALB/c mice bearing 4T1 syngeneic mammary cancer. Animals were pretreated with DEX (s.c., $0.1 \mathrm{mg} / \mathrm{day} / \mathrm{mouse}$ for 5 days) or saline (as controls) and given ADR at a single i.v. dose of $10 \mathrm{mg} / \mathrm{kg}\left(30 \mathrm{mg} / \mathrm{m}^{2}\right)$. Tumor samples were collected at various times up to $24 \mathrm{~h}$. mRNA levels of three cytokines, TNF $\alpha$ (A), IL-1ß (B) and VEGF (C), were determined by quantitative real-time RT-PCR. Time points relate to time after ADR administration.

after ADR administration (Fig. 3A). ADR alone increased the expression of IL-1ß mRNA at $4 \mathrm{~h}$ after ADR administration, but the value returned to normal at $24 \mathrm{~h}$. Pretreatment with DEX reduced IL-1 mRNA expression at all three time points (Fig. 3B). In the absence of DEX, VEGF expression 
A

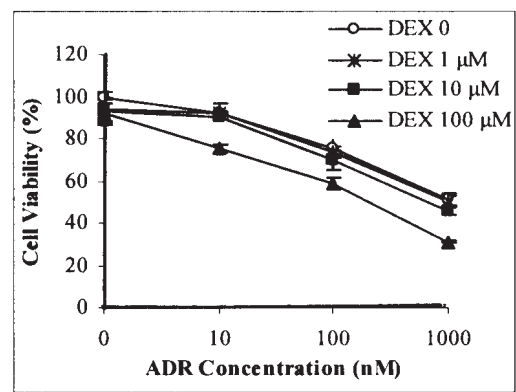

B

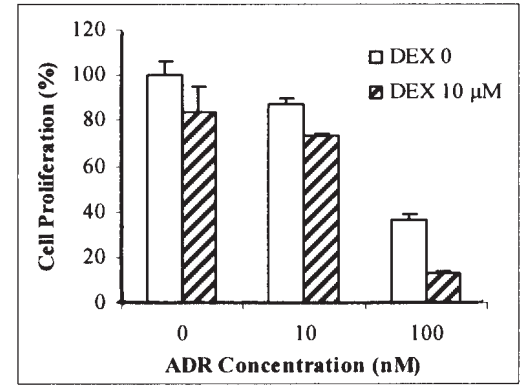

C

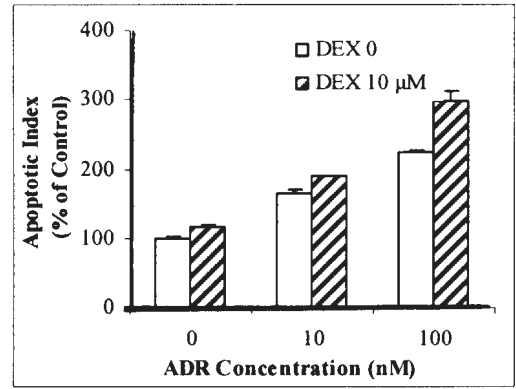

Figure 4. Effects of DEX pretreatment on ADR-induced cell viability, proliferation, and apoptosis for the 4T1 cell line. (A) Cell viability: 4T1 cells were seeded on 96-well microtiter plates (500-1,000 cells/well) and pretreated with gradient concentrations of DEX for $24 \mathrm{~h}$. Cells were then exposed to ADR at gradient concentrations for $24 \mathrm{~h}$. Cell viability percentages were determined by the MTT assay for cell viability. Data were obtained from triplicates of each experiment. (B) Cell proliferation rate: following a similar treatment protocol, the cell proliferation rate was determined by BrdU. Data were obtained from duplicates of each experiment. (C) Apoptosis: following a similar treatment protocol, cells in early and late stages of apoptosis were detected with an annexin V-FITC apoptosis detection kit. was enhanced at $24 \mathrm{~h}$ after ADR administration. With DEX pretreatment, the expression was enhanced at $4 \mathrm{~h}$, but the level returned to normal at $24 \mathrm{~h}$ after ADR treatment, reversing the ADR-induced effects on VEGF expression (Fig. 3C).

Pretreatment with DEX enhances ADR-induced cell death and apoptosis, and inhibits proliferation in cultured cells. In a concentration-dependent manner, ADR alone decreased cell viability; the $\mathrm{IC}_{50}$ value was approximately $1 \mu \mathrm{M}$. Pretreatment with DEX contributed to the effects of ADR. At a DEX concentration of $100 \mu \mathrm{M}$, the $\mathrm{IC}_{50}$ for ADR decreased to about $250 \mathrm{nM}$ (Fig. 4A). Prior exposure to DEX at $10 \mu \mathrm{M}$ increased the inhibitory effect of ADR on cell proliferation (Fig. 4B), and increased ADR-induced cell apoptosis (Fig. 4C).

DEX inhibits activation of nuclear $N F \kappa B$, likely by increasing the production of $I \kappa B$. Analysis of Western blots showed that DEX decreased nuclear NFKB in a concentration-dependent manner, whereas cytoplasmic NFkB accumulated. DEX also increased the expression of the $\mathrm{NF \kappa B}$ inhibitory protein, IкB, which is thought to be involved in the biological effects of DEX (Fig. 5A). ADR alone (500 nM) induced nuclear NFאB expression, which was suppressed by prior exposure to DEX (Fig. 5B). Similar results were observed with the NFKBluciferase promoter assay (Fig. 5C). DEX alone inhibited $\mathrm{NF \kappa B}$ activation, and prior exposure to $\operatorname{DEX}(1-100 \mu \mathrm{M})$ suppressed the ADR (500 nM)-induced NFkB activation.

\section{Discussion}

There have been intensive investigations into the concept that inflammation is an essential component in cancer development and progression $(4,14)$. The potential for using anti-inflammatory agents as chemosensitizers and chemoprotectants is promising $(5,15)$. The anti-inflammatory agent, DEX, has been used in clinical cancer therapy, especially for treatment of leukemia and lymphoma during and after chemotherapy (8-11). Our previous data indicate that pre-treatment with DEX results in two distinct anticancer
A

Cytoplasmic NF $\kappa$ B (p65)

Nuclear NF $\kappa$ B (p65)

I $\mathrm{B}$

$\beta$-actin

B

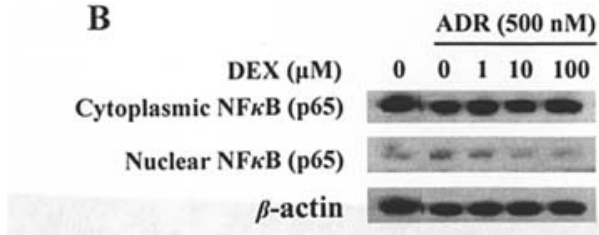

C

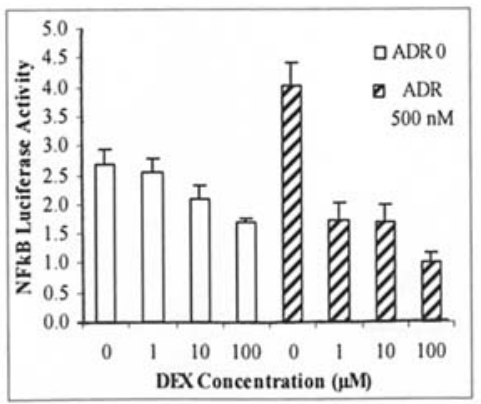

Figure 5. Protein levels of various genes and NFKB-luciferase promoter activity in 4T1 cells treated with DEX alone or in combination with ADR. (A) 4T1 cells were exposed to DEX for $24 \mathrm{~h}$, and cytoplasmic and nuclear proteins were fractionated by nuclear and cytoplasmic extraction reagents. Proteins were analyzed by Western blotting. (B) 4T1 cells were pretreated with DEX for $24 \mathrm{~h}$, followed by the incubation with ADR for $24 \mathrm{~h}$ and cytoplasmic and nuclear proteins were fractionated. (C) 4T1 cells were transfected with an NFkB-luciferase vector for $8 \mathrm{~h}$, then exposed to DEX for $24 \mathrm{~h}$, followed by incubation with ADR or saline (as a control) for an additional $24 \mathrm{~h}$. Cell lysates were collected and tested for NFkB-luciferase activity using the Dual-Luciferase Reporter Assay System. 
effects: reduction of myelosuppression and enhancement of the antitumor activity of chemotherapeutic agents $(12,13)$. These effects are likely to be associated with the fact that DEX modulates the tissue distribution of various drugs by decreasing host tissue uptake (12) and increasing tumor uptake (13) of chemotherapeutic agents such as carboplatin and gemcitabine. DEX also increases the cytotoxicity of these agents in vivo and in vitro $(12,13)$. Since the efficacy data in our previous study were generated in immunocompromised animals (13), it was difficult to examine the involvement of cytokine expression in the DEX-mediated chemosensitization effects. In the present study, we have shown that pretreatment with DEX enhances the antitumor activity of another chemo-therapeutic agent (ADR) in a syngeneic mouse model of breast cancer, demonstrating that the antitumor activity is caused by the modulation of ADR distribution and cytokine expression.

Pro-inflammatory cytokines and chemokines, produced by tumor cells and/or tumor-associated leukocytes and platelets, may contribute directly to tumor development via various mechanisms, including by acting as growth and survival factors, bypassing the p53 tumor suppressor gene, and facilitating angiogenesis and tumor invasion $(4,5,15)$. Our current data demonstrate that DEX pretreatment modulates the ADRassociated pro-inflammatory cytokine profiles as measured by mRNA expression. When evaluated $24 \mathrm{~h}$ after ADR administration, TNF $\alpha$ expression is enhanced and IL-1ß expression is reduced. Expression of VEGF, a marker for angiogenesis, is elevated in most primary and metastatic tumors $(20,21)$. The present results demonstrate that pretreatment of mice with DEX leads to a reduction in the level of VEGF expression at $24 \mathrm{~h}$ after ADR administration (compared to animals treated with ADR alone), indicating that DEX has an anti-angiogenic effect in animals treated with ADR. These results suggest that modulation of cytokine expression might be a key mechanism by which DEX exerts its effects.

Expression of the aforementioned cytokines is believed to be associated with the NFKB signaling pathway $(15,22-25)$. $\mathrm{NF \kappa B}$ is a transcription factor that regulates inflammatory responses by inducing numerous genes, including those coding for cytokines and chemokines (26). Constitutive activation of NFkB, observed in solid tumors (15), appears to support cancer cell survival and to reduce the sensitivity of cells to chemotherapeutic drugs (27). NFkB is also a tumor promoter in inflammation-associated carcinoma, and activation of NFKB constitutes a link between inflammation and cancer (28).

Glucocorticoids, which are potent inhibitors of $\mathrm{NF \kappa B}$, exert their action mainly through glucocorticoid receptordependent induction of the NFאB inhibitory protein, IкB (29). Our current data demonstrate that, in 4T1 cells, DEX

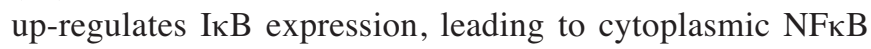
accumulation and reduction of nuclear NFKB activation. Further, expression of IL-1ß and VEGF, two downstream cytokines in this pathway, are indirectly inhibited by DEX.

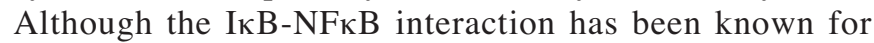
several years, this is the first indication that DEX enhances antitumor activity by inhibiting nuclear NFKB activation.

Our data also show that DEX pretreatment decreases $\mathrm{TNF} \alpha$ production in tumors prior to ADR administration. At
$4 \mathrm{~h}$ after ADR administration, however, DEX increases $\mathrm{TNF} \alpha$ production. $\mathrm{TNF} \alpha$ is suggested to play a dual role in cancer: endogenous $\mathrm{TNF} \alpha$ produced in the tumor microenvironment enhances tumor development and metastasis, but administration of a high dose of $\mathrm{TNF} \alpha$ can induce apoptosis and necrosis of tumor cells (30). Our results suggest that, by inducing $\mathrm{TNF} \alpha$ production upon ADR treatment, DEX pretreatment may make tumor cells more sensitive to ADR-induced apoptosis.

The results from the present study should have an impact on both the use of chemotherapeutic agents, including ADR, and also on the application of DEX as a chemosensitizer and chemoprotectant. In this study, we confirmed the effects of DEX on the pharmacokinetics of chemotherapeutic agents $(12,13)$ using a different class of anticancer agent (ADR). The data generated from the present study suggest that another mechanism may be responsible for the enhancement of the antitumor activity and tumor uptake of chemotherapeutic agents by DEX. Our results showed that DEX increased ADR distribution to tumor tissue. There is also evidence indicating that DEX may exert its effects by reducing the interstitial fluid pressure (IFP) in tumors (31).

Tumor blood vessel walls typically have high permeability to fluids and plasma proteins. In addition, functional lymphatic vessels are absent in tumor tissue (32). When fluid filters from tumor blood vessels into the interstitial space, it can be reabsorbed only slowly into the microvascular network and cannot be taken up by lymphatic vessels. Further, uncontrolled cell proliferation causes tumor cells to become densely packed in the confined environment. All these abnormalities cause fluid accumulation in the interstitial space, directly leading to the elevated IFP throughout the tumor $(33,34)$. The penetration of anti-tumor agents through the interstitial space is a passive process, occurring predominantly by diffusion; interstitial hypertension is believed to be a major physiological barrier to antitumor agent transport. DEX may decrease the IFP by lowering the high vascular permeability of tumor blood vessels (31). From our previous and current work, we postulate that DEX may decrease the elevated volume and pressure in the interstitial space in tumors, allowing a greater concentration of chemotherapeutic agent to accumulate in tumors, thereby improving the antitumor activity of the chemotherapeutic agents. In addition, high IFP in tumor tissues is associated with overexpression of pro-inflammatory cytokines in tumor cells; the effects of DEX on cytokine expression seen in the present study are likely to contribute to increased ADR uptake in tumors by lowering the tumor IFP. Further studies to determine the relationship among tumor IFP, cytokine expression, $\mathrm{NF \kappa B}$ activation, and drug pharmacokinetics are needed in order to verify this hypothesis.

The ADR-mediated increase in pro-inflammatory cytokine expression may also be associated with its side effects in host tissue and drug resistance in tumor cells. Therefore, DEX pretreatment not only enhances the antitumor activity of ADR by affecting several important inflammatory cytokines in the NFкB pathway, but also that it leads to the protection of normal tissues, especially bone marrow, against the toxicity frequently observed during breast cancer treatment. Although additional mechanistic studies, such as determination of 
the effects of DEX on other inflammatory cytokines and cytokine-producing cells, are needed in order to provide a more complete understanding of the effects of DEX on cancer chemotherapy, the present study provides additional evidence for the clinical use of DEX as a chemosensitizer and chemoprotectant for a variety of cancer chemotherapeutic agents.

In summary, although DEX has been in use for many years, these data indicate that it may be of much greater clinical interest for cancer than was previously thought. We demonstrated in an earlier study that DEX could improve the pharmacokinetics of two chemotherapeutic agents and indicated that it might protect patients from hematotoxicity $(12,13)$. In this study, we have demonstrated: a) that these effects are applicable to another class of chemotherapeutic agents; b) that the increased anti-cancer effects occur through inhibition of inflammatory and angiogenic pathways; and c) that the alteration of the tumor microenvironment may play a major role in the chemosensitizing effects of the drug. Therefore, to provide patients with the greatest benefit, new evaluations of the administration of DEX for cancer therapy should be undertaken.

\section{Acknowledgements}

We thank M. Li, K. Wang, and W. Aldrich for excellent technical assistance, Drs R.B. Diasio, J.J. Rinehart, P.L. Triozzi for helpful discussions, and Dr C. Huang for providing reagents for NFKB assays.

\section{References}

1. Jemal A, Siegel R, Ward E, Murray T, Xu J, Smigal C and Thun MJ: Cancer statistics, 2006. CA Cancer J Clin 56: 106-130, 2006.

2. Mouridsen HT: Systemic therapy of advanced breast cancer. Drugs 44: 17-28, 1992.

3. Levin M, Durgam S and Novetsky A: Cyclophosphamide, doxorubicin, and gemcitabine combination chemotherapy for treatment of metastatic and locally advanced breast cancer. Cancer Invest 20: 872-875, 2002.

4. Coussens LM and Werb Z: Inflammation and cancer. Nature 420: 860-867, 2002 .

5. Balkwill $\mathrm{F}$ and Mantovani A: Inflammation and cancer: back to Virchow? Lancet 357: 539-545, 2001.

6. Aapro MS: Present Role of Corticosteroid as Antiemetics. Vol. 121. Springer Verlag, Berlin, 1991.

7. Rutz HP: Effects of corticosteroid use on treatment of solid tumors. Lancet 360: 1969-1970, 2002.

8. Hoelzer D, Ludwig WD, Thiel E, et al: Improved outcome in adult B-cell acute lymphoblastic leukemia. Blood 87: 495-508, 1996.

9. Reiser M, Schnell R, Straub G, Borchmann P, Wilhelm M, Ubelacker R, Wormann B, Munch R, Diehl V and Engert A: DIZE (dexamethasone, idarubicin, and continuous infusion of ifosfamide and etoposide): an effective and well-tolerated new regimen for patients with relapsed lymphoma. Leuk Lymphoma 31: 359-366, 1998.

10. The Italian Group for Antiemetic Research: Dexamethasone alone or in combination with ondansetron for the prevention of delayed nausea and vomiting induced by chemotherapy. N Engl J Med 342: 1554-1559, 2000.

11. Mahesh R, Perumal RV and Pandi PV: Cancer chemotherapyinduced nausea and vomiting: role of mediators, development of drugs and treatment methods. Pharmazie 60: 83-96, 2005.

12. Wang H, Li M, Rinehart JJ and Zhang R: Dexamethasone as a chemoprotectant in cancer chemotherapy: hematoprotective effects and altered pharmacokinetics and tissue distribution of carboplatin and gemcitabine. Cancer Chemother Pharmacol 53: 459-467, 2004.
13. Wang H, Li M, Rinehart JJ and Zhang R: Pretreatment with dexamethasone increases antitumor activity of carboplatin and gemcitabine in mice bearing human cancer xenografts: in vivo activity, pharmacokinetics, and clinical implications for cancer chemotherapy. Clin Cancer Res 10: 1633-1644, 2004.

14. Castro-Caldas M, Mendes AF, Carvalho AP, Duarte CB and Lopes MC: Dexamethasone prevents interleukin-1beta-induced nuclear factor-kappaB activation by up-regulating IkappaBalpha synthesis, in lymphoblastic cells. Mediators Inflamm 12: 37-46, 2003.

15. Bharti AC and Aggarwal BB: Nuclear factor-kappa B and cancer: its role in prevention and therapy. Biochem Pharmacol 64: 883-888, 2002.

16. Zhang Z, Li M, Wang H, Agrawal S and Zhang R: Antisense therapy targeting MDM2 oncogene in prostate cancer: effects on proliferation, apoptosis, multiple gene expression, and chemotherapy. Proc Natl Acad Sci USA 100: 11636-11641, 2003.

17. Li M, Zhang Z, Hill D, Chen X, Wang $\mathrm{H}$ and Zhang R: Genistein, a dietary isoflavone, down-regulates the MDM2 oncogene at both transcriptional and posttranslational levels. Cancer Res 65: 8200-8208, 2005.

18. Wang H, Yu D, Agrawal S and Zhang R: Experimental therapy of human prostate cancer by inhibiting MDM2 expression with novel mixed-backbone antisense oligonucleotides: in vitro and in vivo activities and mechanisms. Prostate 54: 194-205, 2003.

19. Cox SK, Wilke AV and Frazier D: Determination of adriamycin in plasma and tissue biopsies. J Chromatogr 564: 322-329, 1991.

20. Kitajima S, Liu E, Morimoto M, Koike T, Yu Y, Watanabe T, Imagawa $S$ and Fan J: Transgenic rabbits with increased VEGF expression develop hemangiomas in the liver: a new model for Kasabach-Merritt syndrome. Lab Invest 85: 1517-1527, 2005.

21. Kim LS, Huang S, Lu W, Lev DC and Price JE: Vascular endothelial growth factor expression promotes the growth of breast cancer brain metastases in nude mice. Clin Exp Metastasis 21: 107-118, 2004.

22. Altinoz MA and Korkmaz R: NF-kappaB, macrophage migration inhibitory factor and cyclooxygenase-inhibitions as likely mechanisms behind the acetaminophen- and NSAID-prevention of the ovarian cancer. Neoplasma 51: 239-247, 2004.

23. Machuca C, Mendoza-Milla C, Cordova E, Mejia S, Covarrubias L, Ventura J and Zentella A: Dexamethasone protection from TNF-alpha-induced cell death in MCF-7 cells requires NF-kappaB and is independent from AKT. BMC Cell Biol 7: 9-21, 2006.

24. Celec P: Nuclear factor kappa B-molecular biomedicine: the next generation. Biomed Pharmacother 58: 365-371, 2004.

25. Hermoso MA and Cidlowski JA: Putting the brake on inflammatory responses: the role of glucocorticoids. IUBMB Life 55: 497-504, 2003.

26. Arlt A and Schafer H: NFkappaB-dependent chemoresistance in solid tumors. Int J Clin Pharmacol Ther 40: 336-347, 2002.

27. Lin A and Karin M: NF-кB in cancer: a marked target. Semin Cancer Biol 13: 107-114, 2003.

28. Pikarsky E, Porat RM, Stein I, Abramovitch R, Amit S, Kasem S, Gutkovich-Pyest E, Urieli-Shoval S, Galun E and Ben-Neriah Y: NF-kappaB functions as a tumour promoter in inflammationassociated cancer. Nature 431: 461-466, 2004.

29. Auphan N, Di Donato JA, Rosette C, Helmberg A and Karin M: Immunosuppression by glucocorticoids: inhibition of NF-kB activity through induction of $\mathrm{I}-\kappa \mathrm{B}$ synthesis. Science 270 : 286-290, 1995.

30. Balkwill F: Tumor necrosis factor or tumor promoting factor? Cytokine Growth Factor Rev 13: 135-141, 2002.

31. Kristjansen PE, Boucher Y and Jain RK: Dexamethasone reduces the interstitial fluid pressure in a human colon adenocarcinoma xenograft. Cancer Res 53: 4764-4766, 1993.

32. Leu AJ, Berk DA, Lymboussaki A, Alitalo K and Jain RK: Absence of functional lymphatics within a murine sarcoma: a molecular and functional evaluation. Cancer Res 60: 4324-4327, 2000.

33. Jain RK: Transport of molecules in the tumor interstitium: a review. Cancer Res 47: 3039-3051, 1987.

34. Lammerts E, Roswall P, Sundberg C, Gotwals PJ, Koteliansky VE, Reed RK, Heldin NE and Rubin K: Interference with TGF-B1 and $\beta_{3}$ in tumor stroma lowers tumor interstitial fluid pressure independently of growth in experimental carcinoma. Int J Cancer 102: 453-462, 2002. 